\title{
Fault Prediction Method Based on Data Mining in Semiconductor Test Line
}

\author{
Renhong Yue ${ }^{1, a}$ Yumin $\mathrm{Ma}^{1, \mathrm{~b}}$ Fei Qiao ${ }^{1, \mathrm{c}}$ Xinghao $\mathrm{Wu}^{2, \mathrm{~d}}$ \\ 1. CIMS Research Center, Tongji University, Shanghai (201804, China) \\ 2. AMD Technologies Co. Ltd, Suzhou (215021, China) \\ a. yuerenhong@126.com, b. ymma@tongji.edu.cn ${ }^{\text {c. }}$ fqiao@mail.tongji.edu.cn, d. xh.wu@amd.com
}

\section{Keywords: Fault Prediction, Data Mining, Decision Support, Semiconductor Test}

\begin{abstract}
In semiconductor test system, test equipment all have a period of usage. When the test time of equipment is larger than its period of usage, its fault will occur frequently. This paper will use data mining method to predict the next time point of fault based on the history data related to equipment fault. By this, a method of equipment fault prediction will be put forward, and provide the decision support for semiconductor equipment maintenance.
\end{abstract}

\section{Introduction}

Equipment fault will cause production pause, lower equipment utilization rate and loss resulted from delay in semiconductor test, which bring rather negative effect to the system performance. Commonly, some repairing methods will be used, such as checking equipment periodically, storing the standby units etc. However, on the one hand, these passive repairing approaches will result in a waste of resource; On the other hand, they will not be able to reduce the equipment fault rate effectively in essence. The ideal method dealing with equipment fault is to make use of the preventive maintenance. Before equipment fault occurs, the fault time will be predicted, and measures will be taken to make preventive maintenance to reduce the equipment fault rate, even eliminate the occurrence of fault, which will improve the stability and efficiency of semiconductor test system.

Traditional equipment fault prediction approaches use some algorithms to solve the model and gain the fault time point by building up the equipment maintenance model ${ }^{[1,2]}$. However, the model built up by this method is too simplified impossibly to completely reflect the real features of fault and the change trend of parameters related to fault. Meantime, a large portion of computer system resource will be occupied when the model will be solved so that the time for waiting for output results will be rather long. Data Mining is a new technology, which can research for the valuable and preventive information from the known history information. At present, data mining technology has been applied initially to the prediction of parameters in industrial sites. Rakshani etc ${ }^{[3]}$ have achieved fault predictive function in boiler of power plant by the usage of relationship between the parameters in boiler and status of boiler. Firstly, these parameters and status dataset will be preprocessed, which will be as the input of BP neural network, then neural network will be created, learned and trained. Meidan etc ${ }^{[4]}$ have used the conditional mutual information method to identify the main factors of affecting the Lot Cycle-Time (CT), and applied the method to the selective naïve Bayesian Classifier for determining the set of factors which affect the CT. This paper will use data mining method and search for the test parameters related to equipment fault to predict the equipment fault time and equipment distribution in some given range of related parameters based on the larger portion of history test information and the range of parameters. Fault equipment (key components) should be replaced before the fault time of equipment, which will be used for directing the maintenance dispatch and the formulation of maintenance plan 


\section{Data Mining Method for Equipment Fault Prediction}

Common data mining algorithms: (1) Decision Tree Algorithm. Decision Tree is a tree structure similar to Binary Tree or Forest, in which the attributes of samples will be as the nodes, the value of attributes will be as branch of tree and leaf nodes will present the categories of samples. The algorithms of creation of decision tree contain: ID3, C4.5, CART, CHAID, and it will mainly apply to the classification. (2) Clustering Analysis. Clustering analysis is used for evaluating the similarity among the individuals. It applies to the classification of data based on the spatial geometrical distance. If the distance is shorter, the similarity is closer. (3) Bayesian Classifier. Bayesian Classifier is a simple classification method, which uses the supervisory learning method and effectively deals with the future data for classification by training the samples. (4) Association Rules. Association Rules will search for action model by the analysis of association among the different variables or different individuals. There are important parameters, which contain Support and Confidence. (5) Time Series Analysis. Time Series Analysis will make the prediction of future trend by the analysis of time series values in continuous time points or continuous periods. (6) Neural Network. Neural Network has the memorial and learning function for the input and output of samples, and extends the predictive function for unknown inputs by the simulation of the process of human thinking. Its function commonly uses in the areas of classification, the identification of modes, prediction and etc.

This paper selects the time series algorithm based on the comparison among the functions of data mining algorithms, and predicts the future time point of equipment fault by the time series analysis of history data. Common approaches of time series prediction as follows ${ }^{[5,6]}$ :

Table 1 The algorithms of time series

\begin{tabular}{|c|c|}
\hline Time Series Method & Description \\
\hline \multirow{3}{*}{ Smooth Prediction } & $\begin{array}{l}\text { Mobile Average: Gaining the average of } \mathrm{N} \text { recent time series data, and } \\
\text { making the average to be as next data. }\end{array}$ \\
\hline & $\begin{array}{l}\text { Weighted Mobile Average: Assigning the weight of different observed } \\
\text { values by the length of time, gaining the average of } \mathrm{N} \text { recent time } \\
\text { series data, and making the average to be as next data. }\end{array}$ \\
\hline & $\begin{array}{l}\text { Exponential Smooth: Gaining the exponential average by the } \\
\text { exponential } \\
\text { average method, making the average to be as next data. }\end{array}$ \\
\hline $\begin{array}{l}\text { Trend Projective } \\
\quad \text { Prediction }\end{array}$ & $\begin{array}{l}\text { Gaining the evaluative regressive equation as time passing by the } \\
\text { calculate approaches of evaluative regressive coefficient from history } \\
\text { time series. By this, the predictive value of arbitrary time future point } \\
\text { will be gained. }\end{array}$ \\
\hline \multirow{2}{*}{ Regressive Model } & $\begin{array}{l}\text { In this model, time to be as independent variables, and observed value } \\
\text { to be as dependent values. }\end{array}$ \\
\hline & $\begin{array}{l}\text { In this autoregressive model, the hysteretic observed value to be as } \\
\text { dependent values. }\end{array}$ \\
\hline
\end{tabular}

The selection of prediction method bases on the interpretative function of method, significance of parameter estimate and the prediction capability of model. In this paper, data mining approach will use the first regressive model, and time $t$ will be as the independent value, Tsink test value will be as dependent value. Regressive coefficient will be gained in the process of training of model, and achieve the prediction of subsequent fault time point. 


\section{The Instance of Application}

In a semiconductor company, the head of test equipment HST is the component whose fault occurs frequently, and the fault rate of head occupies the $70 \%-80 \%$ in total equipment fault. Among the head fault of equipment, Tsink failure is common fault. When Tsink test value exceeds the threshold value (for example 0.15), Tsink failure will be determined and the failure head must be replaced. In the industrial site, operators make a Buy-Off test about every week to gain the Tsink value. These test records will be saved in EQ system. Tsink failure will be predicted by time series analysis method for history Buy-Off test records.

The Solution of Fault Prediction Method. This paper will use the history data of Tsink test value gained by MFG test operators, and time series algorithm will be used in MS SQL Server SSAS. In assigned Tsink threshold value, next Tsink failure time and head distribution in given range of Tsink value will be predicted.

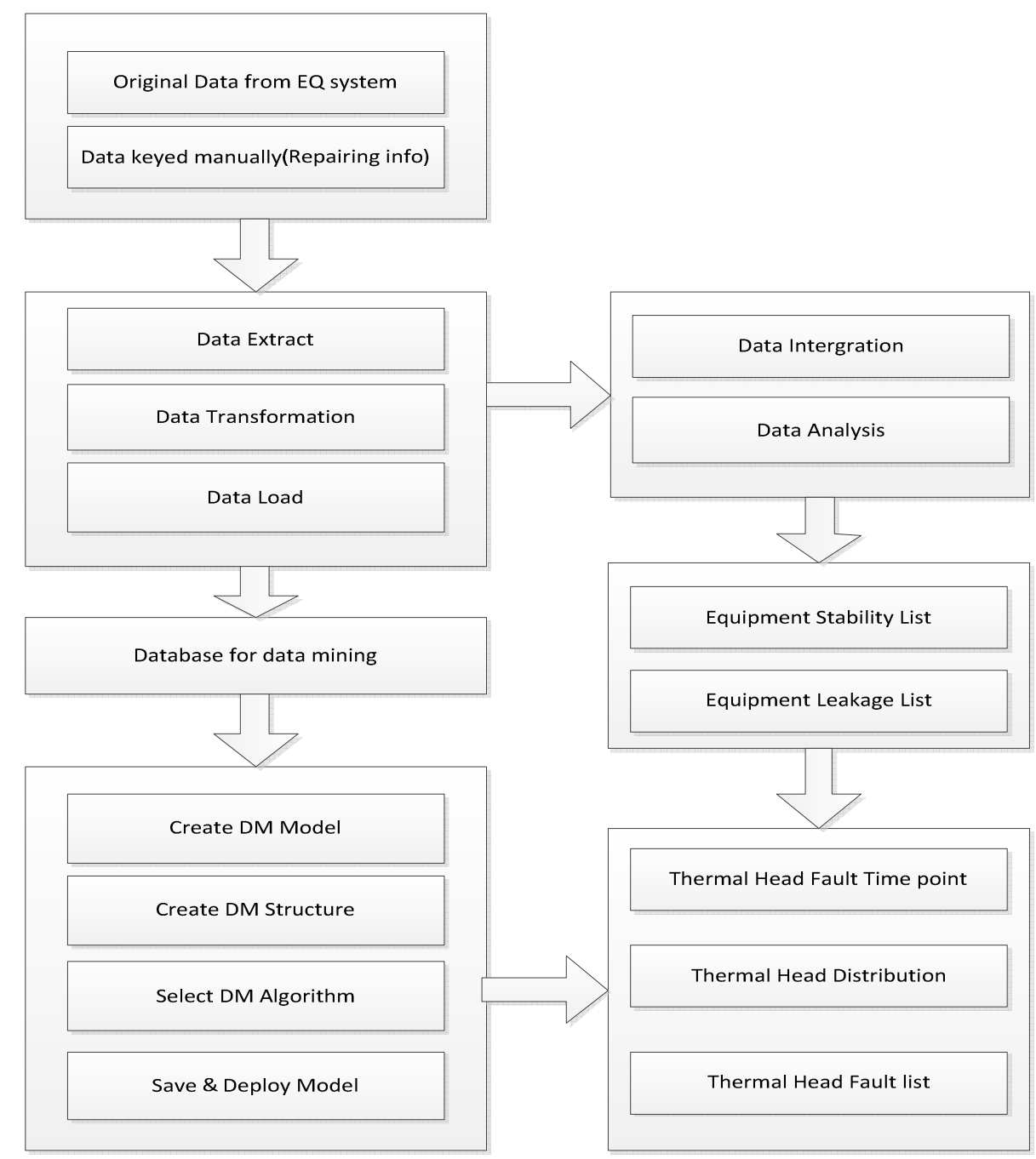

Fig. 1.Solution

The Output of System. The outputs of system contain the judgment of equipment stability and leakage failure based on the mathematics analysis of history data, next predictive time of Tsink fault, the curve of change of Tsink value with time passing and head distribution in a given range of Tsink value. Partial detailed screenshot as follows: 


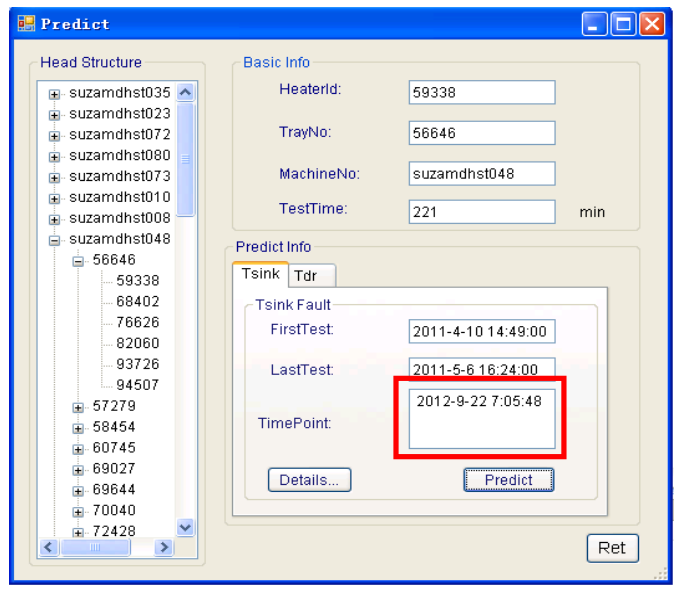

Fig. 2.The predictive time of given head

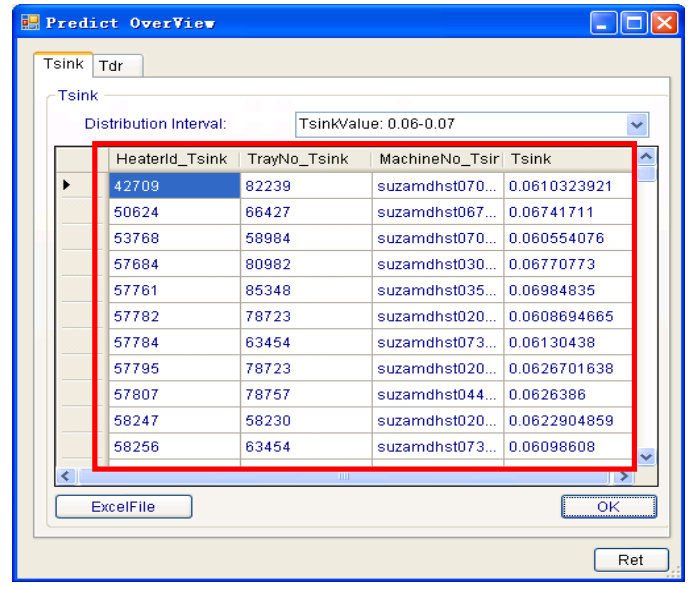

Fig. 3. The distribution of head

The Comparison of Results. The comparison will make between the real time of six heads extracted randomly from repairing room and the preventive time gained by data mining. Detailed comparison as follows:

Table 2 The comparison between real time and predict time

\begin{tabular}{ccc} 
HeadId & Predict Time & Real Time \\
\hline 70911 & $2011-5-24$ & $2011-5-11$ \\
51768 & $2011-5-15$ & $2011-5-5$ \\
55514 & $2011-6-4$ & $2011-5-27$ \\
63962 & $2011-5-19$ & $2011-5-27$ \\
62046 & $2011-6-28$ & $2011-6-12$ \\
83028 & $2011-6-12$ & $2011-6-16$ \\
\hline
\end{tabular}

As shown in the table above, there have been small gaps between real failure time and predictive time. However, there are large gaps between two times. The reasons why exist large gap contain: (1) Because the Tsink value is closely related to pure test time ,if unbalance exist in the front and later time, pure test time will be uneven, and finally cause the preventive result is not so accurate.(2) Time series analysis need a large quantity of basic data to support it . If the number of Tsink records is small, the preventive result will exist big error.

\section{Conclusion}

This paper predicts the next fault time based on the data mining on the history data, and brings data mining technology into fault prediction in semiconductor test line. The system created by this method can be as a function module to integrate into whole enterprise system, and direct the formulation of equipment maintenance plan. However, this method exists several problems: (1) The change trend of Tsink value associates with pure test time of head. If the load of head has a big fluctuation, it will cause the preventive fault time is not so accurate. Thus, it is greatly necessary to keep the load in a constant or reasonable range; (2) Time series analysis need a large quantity of basic data to support it. The number of Tsink records is so smaller that the preventive result will exist big error; (3) Time series has not memory and mode identification function, which just bases on the mathematics statistics of data. Consequent predict model may improve by the usage of Artificial Neural Network or expert system, and the modes (repairing ways) should be classified. If fault mode is determined beforehand, head fault will be predicted, which will not need a large quantity of history data to support. 
The paper is supported by Project of National Natural Science Foundation of China (61034004); Project of Shanghai Science and Technology Commission (10dz1120110, 09DZ1120600, 11ZR1440400)

\section{References}

[1] Han Bang-jun, Fan Xiu-min, Ma Deng-ze, The optimization of preventive maintenance strategy in limited time regions [J], Shanghai Jiaotong University journal, 2003. (5)

[2] LI-hong, Qian Sheng-san, The optimization of preventive maintenance plan in clustering semiconductor manufacture equipment, Manufacture Technology, 2005. (11)

[3] ElyasRakhshani, Iman Sariri, Kumars Rouzbehi, Application of Data Mining on Fault Detection and Prediction in Boiler of Power Plant Using Artificial Neural Network, POWERENG 2009 Lisbon, Portugal, March 18-20, 2009.

[4] Yair Meidan, Boaz Lerner, Gad Rabinowitz, and Michael Hassoun, Cycle-Time Key Factor Identification and Prediction in Semiconductor Manufacturing Using Machine Learning and Data Mining, IEEE TRANSACTIONS ON SEMICONDUCTOR MANUFACTURING, VOL. 24, NO. 2, MAY 2011.

[5] Xie Bang-chang, Business Intelligence and Data Mining in Microsoft SQL Server, Machinery Industrial Press, 2008.

[6] Yang Shu-zi, WU-Ya, Engineering Application on Time Series(I,II), Huazhong University of Science and Technology Press, 1991. 